Diabetologe 2014 · 10:196-197

DOI 10.1007/s11428-014-1227-3

Online publiziert: 8. Mai 2014

๑) Springer-Verlag Berlin Heidelberg 2014
W.A. Scherbaum

Universitätsklinikum, Heinrich-Heine-Universität, Düsseldorf

\section{Zehn Jahre Der Diabetologe}

\section{Erfolg mit vielen Vätern}

Das 10-jährige Bestehen der Zeitschrift Der Diabetologe ist ein willkommener Anlass, die bisherige Entwicklung der Zeitschrift zu beleuchten und einen Blick nach vorn zu richten.

Die anfängliche Frage, ob es wünschenswert ist und ob es sich für die Ärzteschaft lohnt, eine deutschsprachige Zeitschrift zum Thema Diabetologie zu gründen, wurde rasch beantwortet. Das Konzept der Zeitschrift basierte auf dem vielfach geäußerten Wunsch, ein speziell auf das Thema Diabetes ausgerichtetes Fortbildungsjournal anzubieten, in dem die Neuerungen und Forschungsergebnisse der wichtigsten Gebiete der Diabetologie von Experten übersichtlich dargestellt und bezüglich ihrer klinischen Relevanz bewertet werden. Dies wird inzwischen unterstützt durch die Einbindung von wichtigen Beiträgen der in jedem Frühjahr stattfindenden DiabetesUpdate-Veranstaltungen, bei denen das in den vergangenen 1 bis 2 Jahren hinzugewonnene Wissen aktuell aufgearbeitet und diskutiert wird.

> Schon 2007 wurde Der Diabetologe in der Shortlist der Deutschen Fachpresse als beste Fachzeitschrift des Jahres ausgezeichnet.

Im Jahr 2009 gelangen die Aufnahmen in das „Institute for Scientific Information“ (ISI) und damit in die Datenbank Web of Science sowie in die Journal Citation Reports (JCR).

Auf diese Leistung dürfen alle Beteiligten stolz sein, zumal es damit einem deutschsprachigen Journal gelungen ist, sich in diesem überwiegend englischsprachigen internationalen Kontext zu platzieren. Dafür danke ich allen Mitgliedern der Schriftleitung: Dr. R.A. Bierwirth, Prof. Dr. M. Blüher, Prof Dr. Dr. h.c. H. Drexel, Prof. Dr. R. Landgraf, Prof. Dr. R. Lehmann, Dr. A. Lueg, Prof. Dr. S. Martin, Prof. Dr. M. Roden, Prof. Dr. G.A. Spinas und Prof. Dr. T. Stulnig. Außerdem danke ich den Mitgliedern des Wissenschaftlichen Beirats für die Kooperation und insbesondere den zahlreichen Autoren, die ihre Spuren in der Zeitschrift Der Diabetologe hinterlassen haben. Dank gebührt an dieser Stelle auch den zahlreichen Reviewern, die für die Leserschaft im Verborgenen bleiben, aber mit ihrer altruistischen Arbeit einen großen Beitrag für die Wissenschaftsgemeinschaft leisten.

In Der Diabetologe wurden von Anfang an neben den mehr wissenschaftlich orientierten klinischen Übersichtsreferaten auch Continuing-Medical-Education(CME)-Beiträge angeboten, in denen besondere Themen auf Fortbildungsebene dargestellt werden, für die zertifizierte CME-Punkte erworben werden können. Diese Rubrik liegt uns ganz besonders am Herzen, zumal wir damit ganz konkrete Hinweise und Handlungsempfehlungen für diagnostische sowie therapeutische Prozeduren geben können, die für die ärztliche Praxis relevant sind. Um diese Rubrik hat sich Herr Prof. R. Landgraf als deren Herausgeber besonders verdient gemacht. Mit seiner großen Erfahrung, aber auch mit seiner überzeugenden Kritik versteht er es immer wieder, im stetigen Bestreben um die optimale Aufarbeitung und Präsentation des Themas die Autoren der CME-Beiträge zu motivieren und sie damit im Sinne unserer Leser zu Höchstleistungen zu treiben.

Daneben findet der Leser in der Zeitschrift Referate zu aktuellen Publikationen. An dieser Stelle soll den zahlreichen jungen Wissenschaftlern gedankt werden, die diese Publikationen für unsere Leserschaft gut verständlich aufbereiten und die medizinischen Aussagen auf den Punkt bringen. Um diese wichtige $\mathrm{Ru}$ brik „Journal Club“ kümmert sich Herr Prof. S. Martin, der mit seiner umfangreichen Erfahrung die Autoren auswählt und die Nachrichten kritisch prüft. Für dieses große Engagement gebührt ihm auch der Dank der Leserschaft.

Eine Reihe weiterer Angebote wie Kurznachrichten und Mitteilungen sowie Beiträge verschiedener kooperierender Fachgesellschaften und Fachverbände bereichert unsere Zeitschrift. Dies sind die Deutsche Gesellschaft für Innere Medizin (DGIM), die Deutsche Diabetes-Stiftung (DDS), der Bund Deutscher Internisten (BDI), der Bund Deutscher Endokrinologen (BDE), der Bundesverband Deutscher Diabetes-Kliniken (BVDK), die Arbeitsgemeinschaft Niedergelassener Diabetologen (AND) sowie der Verband der Diabetes-Beratungs- und Schulungsberufe in Deutschland (VDBD). Aus dieser Liste geht auch hervor, dass sich Der Diabetologe nicht nur an Diabetologen richtet, sondern dass es unser Bestreben ist, mit der Zeitschrift viele Kollegen zu erreichen, die z. T. primär einen anderen Fokus haben, aber Diabetespatienten mitbetreuen. Dies kommt unserem Wunsch und Ziel entgegen, die Grundlinien der evidenz- 
basierten Diabetologie in einem breiteren Kontext von Fachgebieten zu vermitteln.

Schließlich und nicht zuletzt möchte ich allen Beteiligten des Springer-Verlags für die ausgesprochene Unterstützung danken, die wir bei der täglichen Arbeit mit der Zeitschrift erfahren. Über den Vertrieb der Print-Version hinaus ist Der Diabetologe elektronisch derzeit weltweit in mehr als 7600 Instituten und Bibliotheken im Volltext verfügbar. Damit erreichen wir eine Leserschaft, die weit über die gedruckten Exemplare hinausgeht und uns weiterhin zu höchsten Leistungen verpflichten wird. In diesem Sinne streben wir nach weiteren Verbesserungen unserer Zeitschrift und sind für die Kritik und Anregungen unserer Leser dankbar.

\section{loere llevban}

Werner A. Scherbaum

Chefredakteur

\section{Korrespondenzadresse}

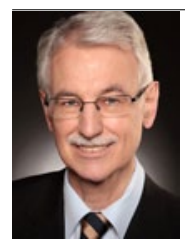

Prof. Dr. W.A. Scherbaum

Universitätsklinikum,

Heinrich-Heine-Universität

Moorenstr. 5

40225 Düsseldorf

scherbaum@uni-duesseldorf.de

\section{Einhaltung ethischer Richtlinien}

Interessenkonflikt. W.A. Scherbaum gibt an, dass kein Interessenkonflikt besteht.

\section{Diabetes und Adipositas häufiger in sozioökonomisch benachteiligten Regionen}

In einer sozioökonomisch benachteiligten Region zu leben, ist ein Risikofaktor dafür, von Diabetes mellitus und Adipositas (Fettleibigkeit) betroffen zu sein. Dies gilt auch unabhängig vom individuellen Sozial- und Bildungsstatus der Bewohner. Zu diesem Schluss kommen Wissenschaftler vom Institut für Gesundheitsökonomie und Management im Gesundheitswesen (IGM) am Helmholtz Zentrum München (HMGU) und der Abteilung für Epidemiologie und Gesundheitsmonitoring am Robert Koch-Institut (RKI) in Berlin.

Ausgewertet wurden die Daten von über 33.000 Personen im Alter ab 30 Jahren, die 2009 und 2010 an den telefonischen Gesundheitsbefragungen „Gesundheit in Deutschland Aktuell (GEDA)" des RKI teilgenommen hatten.

In sozioökonomisch benachteiligten Regionen leiden die Bewohner überdurchschnittlich häufig an Diabetes und Übergewicht. Die geographische Benachteiligung wird als „regionale Deprivation“ bezeichnet. Ermittelt wird sie anhand des „,German Index of Multiple Deprivation“ (GIMD), der aus regional verfügbaren Informationen wie Einkommen, Beschäftigung, Bildung und Sicherheit in einem definierten Gebiet gebildet wird. Neben dem GIMD wurden in der Datenanalyse auch individuelle Risikofaktoren wie Alter, Geschlecht, Body-Mass-Index, Rauchen, körperliche Bewegung und Bildung berücksichtigt.

In Regionen mit der höchsten Benachteiligung (Deprivation) lag die Häufigkeit eines Typ-2-Diabetes bei 8,6 Prozent der Befragten und für Adipositas bei 16,9 Prozent, gegenüber 5,8 bzw. 13,7 Prozent der Befragten in nur gering benachteiligten Regionen. Diese Ergebnisse wurden auf relevante Unterschiede in allen individuellen Faktoren überprüft. Verglichen mit Männern und Frauen in den am wenigsten benachteiligten Regionen hatten Personen in den Gebieten mit der höchsten Deprivation eine rund 20\% höhere Wahrscheinlichkeit, an Typ-2-Diabetes erkrankt zu sein, und eine um fast $30 \%$ erhöhte Wahrscheinlichkeit für Adipositas. Hohe regionale Deprivation war insbesondere bei Frauen ein unabhängiger Einflussfaktor für das Auftreten von Diabetes und Adipositas.
Bei Männern ließ sich nur ein statistisch signifikanter und unabhängiger Zusammenhang für Adipositas nachweisen.

Die Ergebnisse weisen auf die Bedeutung regionaler Faktoren im Zusammenhang mit häufigen Gesundheitsproblemen in Deutschland hin. Dabei sind räumliche Risikofaktoren wie materielle und soziale Deprivation ein wichtiger Ansatzpunkt, um regionalspezifische, effektive Präventionsmaßnahmen zu erarbeiten. Für ganzheitliche Präventionsstrategien sollten regionale und individuelle Risikofaktoren identifiziert und deren Interaktion beleuchtet werden.

Literatur: Maier W, Scheidt-Nave C, Holle R, Kroll LE, Lampert T, Du Y, Heidemann C, Mielck A (2014) Area level deprivation is an independent determinant of prevalent type 2 diabetes and obesity at the national level in Germany. Results from the national telephone health interview surveys 'German Health Update' GEDA 2009 and 2010. PLOS ONE. doi: 10.1371/journal.pone.0089661

Quelle: Robert-Koch-Institut, www.rki.de 